Urologe $2017 \cdot 56: 364-366$

DOI 10.1007/s00120-017-0327-2

Online publiziert: 1. Februar 2017

(c) Der/die Autor(en) 2017. Dieser Artikel ist eine Open-Access-Publikation.

CrossMark

\author{
J. F. Alidjanov ${ }^{1}$ A. Pilatz ${ }^{2}$. U. A. Abdufattaev ${ }^{3} \cdot$ J. Wiltink $^{4} \cdot$ W. Weidner ${ }^{2}$. \\ K. G. Naber ${ }^{5,6} \cdot$ F. Wagenlehner ${ }^{2}$ \\ ' Department of Outpatient Urology, JSC Republican Specialized Center of Urology, Tashkent, Usbekistan \\ ${ }^{2}$ Klinik und Poliklinik für Urologie, Kinderurologie und Andrologie, Justus-Liebig-Universität Gießen, \\ Universitätsklinikum Gießen und Marburg $\mathrm{GmbH}$, Gießen, Deutschland \\ ${ }^{3}$ Department of Urology, Tashkent Medical Academy, Tashkent, Usbekistan \\ ${ }^{4}$ Klinik und Poliklinik für Psychosomatische Medizin und Psychotherapie, Johannes Gutenberg- \\ Universität, Mainz, Deutschland \\ ${ }^{5}$ Technische Universität München, München, Deutschland \\ ${ }^{6}$ Straubing, Deutschland
}

\title{
Neuer Fragebogen zur deutschen Validierung des „Acute Cystitis Symptom Score"
}

namik“"war jedoch suboptimal, was auf eine möglicherweise missverständliche Formulierung der 2. und 3. Frage zu erklären war [1]. Deshalb haben die Autoren den urheberrechtlich geschützten ACSS in allen sprachlichen Versionen entsprechend überarbeitet. Die revidierte deutsche Version des ACSS mit der überarbeiteten Rubrik „Dynamik“ und weiteren sprachlichen Präzisierungen steht jetzt für den Einsatz in Praxis und Klinik zur Verfügung (• Abb. 1 und 2).

\section{Korrespondenzadresse}

apl. Prof. Dr.med. Dr.h.c. K. G. Naber

Karl-Bickleder-Str. 44c, 94315 Straubing,

Deutschland

kurt@nabers.de

en. Ein Summenscore von $\geq 6$ Punkten der Kategorie „Typische Symptome“ war hochsignifikant mit einer akuten Zystitis assoziiert (Sensitivität 94,7\%, Spezifität $82,4 \%$ ). Die Auswertungen der klinischen Validierung der usbekischen und russischen Versionen des ACSS (Teil B, - Abb. 2) ergaben als patientenberichtetes Ergebnis („patient-reported outcome“) eine sehr gute Trennschärfe zwischen Erfolg und Nicht-Erfolg einer durchgeführten Therapie für den Summenscore in der Kategorie "Typische Symptome" mit oder ohne Heranziehung der Antworten auf Fragen zur Lebensqualität. Die Trennschärfe zum Behandlungserfolg in der Rubrik „Dy-
Hinweis. Der ACSS ist urheberrechtlich geschützt mit

a) Certificate of Deposit of Intellectual Property in Fundamental Library of Academy of Sciences of the Republic of Uzbekistan, Tashkent (Registration number 2463; August 26, 2015) [http://avtor-web. com/index.php?option=com_desposition\& task=display_desp_det\&id=2612\&lang=ru] b) Certificate of the International Online Copyright Office, European Depository, Berlin, Germany (Nr. EU01-000764; October 21, 2015) [http://inter.interoco. com/index.php?option=com_desposition\& task=display_desp_det\&id=1013\&lang=en]

Interessenkonflikt. J.F. Alidjanov, A. Pilatz, U.A. Abdufattaev, J. Wiltink, W. Weidner, K.G. Naber und F. Wagenlehner geben an, dass kein Interessenkonflikt besteht.
Open Access. Dieser Artikel wird unter der Creative Commons Namensnennung 4.0 International Lizenz (http://creativecommons.org/licenses/by/4.0/deed. de) veröffentlicht, welche die Nutzung, Vervielfältigung, Bearbeitung, Verbreitung und Wiedergabe in jeglichem Medium und Format erlaubt, sofern Sie den/die ursprünglichen Autor(en) und die Quelle ordnungsgemäßnennen, einen Linkzur Creative Commons Lizenz beifügen und angeben, ob Änderungen vorgenommen wurden.

\section{Literatur}

1. Alidjanov JF, Abdufattaev UA, Makhsudov SA, Pilatz A, Akilov FA, Naber KG, Wagenlehner FME (2016) The acute cystitis symptom score for patient-reported outcome assessment. Urol Int 97(4):402-409 


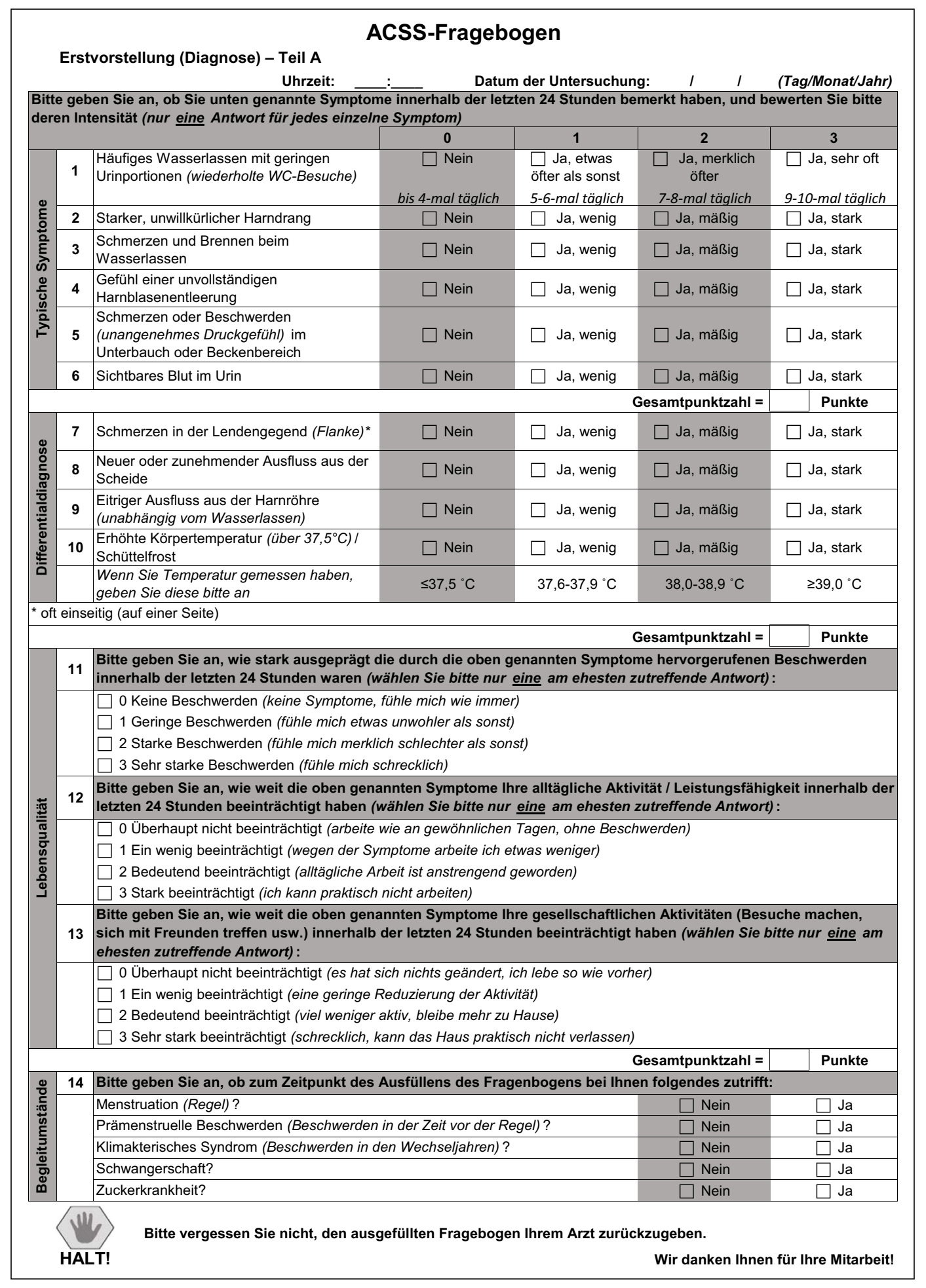

Abb. $1<$ Deutsche Version des ACSS-Fragebogens: Teil A (Erstvorstellung) zur klinischen Diagnostik und Differentialdiagnostik der akuten unkomplizierten Zystitis bei Frauen 


\section{Kommentare}

Kontrollvorstellung (Folgebefund) - Teil B

\section{ACSS-Fragebogen}

Uhrzeit:

Datum der Untersuchung: $\quad l \quad l \quad$ (Tag/Monat/Jahr)

Bitte geben Sie an, ob Sie irgendwelche Änderungen in Ihrem Zustand seit dem Ausfüllen des ersten Teils unseres

Fragebogens bemerkt haben? (kreuzen Sie bitte Ihre Antwort an):

$\square 0$ Ich fühle mich jetzt ausgezeichnet (alle Symptome sind endgültig vergangen)

$\square 1$ Mir geht es jetzt wesentlich besser (die Mehrheit der Symptome sind vergangen)

$\square 2$ Ich fühle mich jetzt nur gering besser (die Mehrheit der Symptome sind immer noch da)

$\square$ Es gibt jetzt keine Änderung meines Zustands (alle Symptome sind noch vorhanden)

$\square 4$ Es ist jetzt schlimmer geworden (mein Zustand hat sich verschlechtert)

Bitte geben Sie an, ob Sie unten genannte Symptome innerhalb der letzten 24 Stunden bemerkt haben, und bewerten Sie bitte

deren Intensität (nur eine Antwort für jedes einzelne Symptom) :

\begin{tabular}{|c|c|c|c|c|c|c|}
\hline & 0 & 1 & 2 & 3 \\
\hline \multirow{7}{*}{ 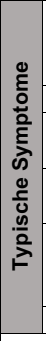 } & 1 & $\begin{array}{l}\text { Häufiges Wasserlassen mit geringen } \\
\text { Urinportionen (wiederholte WC-Besuche) }\end{array}$ & $\square$ Nein & $\begin{array}{l}\square \text { Ja, etwas } \\
\text { öfter als sonst }\end{array}$ & $\begin{array}{l}\square \text { Ja, merklich } \\
\text { öfter }\end{array}$ & $\square$ Ja, sehr oft \\
\hline & & & bis 4-mal täglich & 5-6-mal täglich & 7-8-mal täglich & 9-10-mal täglich \\
\hline & 2 & Starker, unwillkürlicher Harndrang & $\square$ Nein & $\square$ Ja, wenig & $\square \mathrm{Ja}$, mäßig & $\square$ Ja, stark \\
\hline & 3 & Schmerzen und Brennen beim Wasserlassen & $\square$ Nein & $\square$ Ja, wenig & $\square$ Ja, mäßig & $\square$ Ja, stark \\
\hline & 4 & $\begin{array}{l}\text { Gefühl einer unvollständigen } \\
\text { Harnblasenentleerung }\end{array}$ & $\square$ Nein & $\square$ Ja, wenig & $\square$ Ja, mäßig & $\square$ Ja, stark \\
\hline & 5 & $\begin{array}{l}\text { Schmerzen oder Beschwerden } \\
\text { (unangenehmes Druckgefühl) im Unterbauch } \\
\text { oder Beckenbereich }\end{array}$ & $\square$ Nein & $\square$ Ja, wenig & $\square$ Ja, mäßig & $\square$ Ja, stark \\
\hline & 6 & Sichtbares Blut im Urin & $\square$ Nein & $\square$ Ja, wenig & $\square$ Ja, mäßig & $\square$ Ja, stark \\
\hline \multicolumn{6}{|c|}{ Gesamtpunktzahl = } & \begin{tabular}{l|l} 
& Punkte \\
\end{tabular} \\
\hline \multirow{5}{*}{ 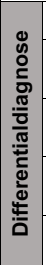 } & 7 & Schmerzen in der Lendengegend (Flanke) * & $\square$ Nein & $\square$ Ja, wenig & $\square$ Ja, mäßig & $\square$ Ja, stark \\
\hline & 8 & $\begin{array}{l}\text { Neuer oder zunehmender Ausfluss aus der } \\
\text { Scheide }\end{array}$ & $\square$ Nein & $\square$ Ja, wenig & $\square$ Ja, mäßig & $\square$ Ja, stark \\
\hline & 9 & $\begin{array}{l}\text { Eitriger Ausfluss aus der Harnröhre } \\
\text { (unabhängig vom Wasserlassen) }\end{array}$ & $\square$ Nein & $\square$ Ja, wenig & $\square$ Ja, mäßig & $\square$ Ja, stark \\
\hline & 10 & $\begin{array}{l}\text { Erhöhte Körpertemperatur (über } 37,5^{\circ} \mathrm{C} \text { ) / } \\
\text { Schüttelfrost }\end{array}$ & $\square$ Nein & $\square$ Ja, wenig & $\square$ Ja, mäßig & $\square$ Ja, stark \\
\hline & & $\begin{array}{l}\text { Wenn Sie Temperatur gemessen haben, } \\
\text { geben Sie diese bitte an }\end{array}$ & $\leq 37,5^{\circ} \mathrm{C}$ & $37,6-37,9^{\circ} \mathrm{C}$ & $38,0-38,9^{\circ} \mathrm{C}$ & $\geq 39,0{ }^{\circ} \mathrm{C}$ \\
\hline
\end{tabular}
* oft einseitig (auf einer Seite)

Gesamtpunktzahl = $\quad$ Punkte

11 Bitte geben Sie an, wie stark ausgeprägt die durch die oben genannten Symptome hervorgerufenen Beschwerden

11 innerhalb der letzten 24 Stunden waren (wählen Sie bitte nur eine am ehesten zutreffende Antwort):

0 Keine Beschwerden (keine Symptome, fühle mich wie immer)

1 Geringe Beschwerden (fühle mich etwas unwohler als sonst)

2 Starke Beschwerden (fühle mich merklich schlechter als sonst)

$\square$ Sehr starke Beschwerden (fühle mich schrecklich)

12 Bitte geben Sie an, wie weit die oben genannten Symptome Ihre alltägliche Aktivität / Leistungsfähigkeit innerhalb der

12 letzten 24 Stunden beeinträchtigt haben (wählen Sie bitte nur eine am ehesten zutreffende Antwort):

0 Überhaupt nicht beeinträchtigt (arbeite wie an gewöhnlichen Tagen, ohne Beschwerden)

1 Ein wenig beeinträchtigt (wegen der Symptome arbeite ich etwas weniger)

2 Bedeutend beeinträchtigt (alltägliche Arbeit ist anstrengend geworden)

3 Stark beeinträchtigt (ich kann praktisch nicht arbeiten)

Bitte geben Sie an, wie weit die oben genannten Symptome Ihre gesellschaftlichen Aktivitäten (Besuche machen, sich

13 mit Freunden treffen usw.) innerhalb der letzten 24 Stunden beeinträchtigt haben (wählen Sie bitte nur eine am ehesten zutreffende Antwort):

$\square 0$ Überhaupt nicht beeinträchtigt (es hat sich nichts geändert, ich lebe so wie vorher)

$\square 1$ Ein wenig beeinträchtigt (eine geringe Reduzierung der Aktivität)

$\square 2$ Bedeutend beeinträchtigt (viel weniger aktiv, bleibe mehr zu Hause)

3 Sehr stark beeinträchtigt (schrecklich, kann das Haus praktisch nicht verlassen)

\begin{tabular}{|c|c|c|c|c|}
\hline \multicolumn{4}{|c|}{ Gesamtpunktzahl = } & Punkte \\
\hline \multirow{6}{*}{ 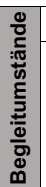 } & 14 & \multicolumn{3}{|c|}{ Bitte geben Sie an, ob zum Zeitpunkt des Ausfüllens des Fragenbogens bei Ihnen folgendes zutrifft: } \\
\hline & & Menstruation (Regel)? & $\square$ Nein & $\square \mathrm{Ja}$ \\
\hline & & Prämenstruelle Beschwerden (Beschwerden in der Zeit vor der Regel)? & $\square$ Nein & $\mathrm{Ja}$ \\
\hline & & Klimakterisches Syndrom (Beschwerden in den Wechseljahren)? & $\square$ Nein & $\mathrm{Ja}$ \\
\hline & & Schwangerschaft? & $\square$ Nein & $\square \mathrm{Ja}$ \\
\hline & & Zuckerkrankheit? & $\square$ Nein & $\square \mathrm{Ja}$ \\
\hline
\end{tabular}

Bitte vergessen Sie nicht, den ausgefüllten Fragebogen Ihrem Arzt zurückzugeben.

HALT!

Wir danken Ihnen für Ihre Mitarbeit!

Abb. $2<$ Deutsche Version des ACSS-Fragebogens: Teil B (Kontrollvorstellung) zum Verlauf/ Behandlungserfolg bei der akuten unkomplizierten Zystitis bei Frauen 